\title{
Capability of the accelerated protons as the origin of white-light emission of solar flare
}

\author{
Kyoko WATANABE* \\ National Defense Academy of Japan, 1-10-20 Hashirimizu, Yokosuka 239-8686, Japan \\ E-mail: kwatanaenda.ac.jp

\section{Satoshi MASUDA} \\ Institute for Space-Earth Environmental Research (ISEE), Nagoya University, Furo-cho, \\ Chikusa-ku, Nagoya 464-8601, Japan
}

\section{Masanori OHNO}

Department of Physical Sciences, Hiroshima University, 1-3-1 Kagamiyama,

Higashi-Hiroshima, Hiroshima 739-8516, Japan

\begin{abstract}
In association with strong solar flares, we sometimes observe enhancements of visible continuum radiation, which is known as a "white-light flare". As most white-light (WL) events show close correlations in time and location with hard X-rays and/or radio emission, there is some consensus that WL emission originates from accelerated particles, especially non-thermal electrons. One model proposes that WL is emitted near the photosphere; however, non-thermal electrons are thermalized in the chromosphere and cannot reach the photosphere. Thus, there is a problem: how can the energy of non-thermal electrons - and/or other accelerated particles such as high-energy protons - propagate to the photosphere and produce WL emission? In the present study, we investigate the possibility that accelerated protons may produce the WL emission of solar flares. We found $51 \mathrm{WL}$ events observed by Hinode/SOT. Among them, gamma-rays with energies greater than $1 \mathrm{MeV}$ were observed only in the X1.8-class flare on October 23, 2012 and in the M7.9-class flare on June 25, 2015. Focusing on these flare events, we compare the energetics of WL emission and of accelerated ion fluxes. We find that it is difficult for accelerated ions to provide sufficient energy to account for WL emission.
\end{abstract}

35th International Cosmic Ray Conference - ICRC2017

10-20 July, 2017

Bexco, Busan, Korea

${ }^{*}$ Speaker. 


\section{Introduction}

In association with solar flares, we sometimes observe enhancements of visible continuum (white-light or WL) radiation. The first white-light flare (WLF) to be discovered - the Carrington flare [四 - was one of the class now known as "super flares". In general, WLFs are mainly associated with energetic events such as GOES X-class flares, and they are still rarely observed. However, using recent high-precision observations from spacecraft (Yohkoh, TRACE, Hinode, etc.), WLFs have been observed in weaker flares such as GOES C-class flares [1], [1, 田, 回].

Although more than 150 years have elapsed since the discovery of WLFs, the mechanism of WL emission is still not fully understood. One of the most well-known correlations with WL emission is the emission of hard X-rays originating from accelerated electrons. Observationally, WL emission is well correlated with hard X-ray and radio emission both in the time profile and

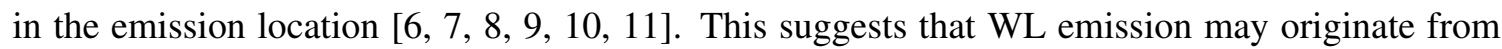
non-thermal electrons. By comparing the WL and hard X-ray emissions, one finds that the energy

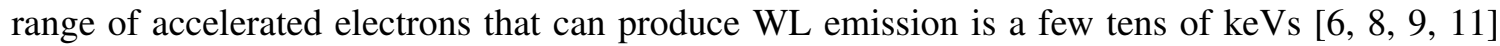
and that the total energy of the observed WL emission is consistent with the total energy of the accelerated electrons that produce the hard X-rays.

However, there is a problem concerning the height of WL emission. Theoretically, one expects WL to be emitted near the photosphere, but non-thermal electrons with energies around $50-100 \mathrm{keV}$ are almost completely thermalized by the time they reach the lower chromosphere. Hard X-rays are also emitted around the lower chromosphere. To reach down to the photosphere, accelerated electrons would need to have energies greater than $900 \mathrm{keV}$ [目]. Even if such highenergy electrons exist, there are not enough of them to explain the total energy of WL emission.

Observationally, the relationship between the emission heights of WL and of hard X-rays has

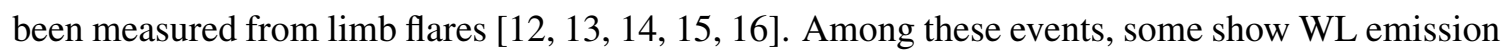

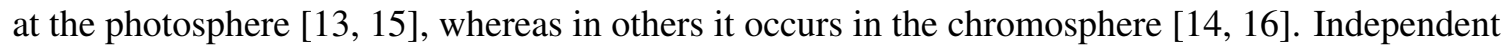
of WL emission height, position differences between the WL and hard X-ray emissions have been observed in some events [14]]. Conversely, other analytical methods show good height correlations [123, [6]]. Determining the height of WL emission is not straightforward and remains an unsolved question.

Alternatively, Neidig [目] proposed a number of energy-transport mechanisms for WLFs, one of which involved high-energy proton beams. If high-energy protons are produced in a solar flare, they can easily reach the photosphere. Theoretically, protons with energies greater than $20 \mathrm{MeV}$ can penetrate to the photosphere [[D], where there is a possibility that they may produce WL emission.

Evidence for ion acceleration that can produce high-energy protons can, in principle, be obtained from solar neutrons and/or line gamma-ray emissions. Solar neutrons are produced by the direct interaction of accelerated ions with the solar atmosphere. They can travel to the Earth [ए]8], but it is not easy to observe them. Usually, solar neutrons are observed by neutron monitors and so-

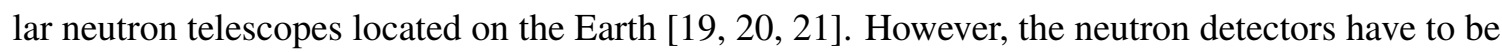
located on the sunlit side of the Earth, and they have to be able to penetrate through the attenuation caused by the Earth's atmosphere to be detected.

Line gamma-rays are also produced by the interaction of accelerated ions with the solar atmosphere, and they can be observed by X-ray and gamma-ray observing satellites. Line gamma-rays, 
which originate from nuclear de-excitation (1-8 MeV) and neutron-capture (2.223 MeV) processes, are observed, along with a continuous bremsstrahlung component. Although line gamma-rays cannot be detected clearly in many events because they are buried in the bremsstrahlung component, we do find some evidence for the existence of a line gamma-ray component.

In this paper, we investigate the ability of accelerated protons to produce the WL emission of solar flares.

\section{Observations and analysis}

\subsection{Event selection}

The event-selection period for this WLF study extended from January 2011 to February 2016. To select flare events, we used the Hinode flare catalogue [[2]], which lists 11,387 flare events in this period. We chose the M- and X-class flares because WLFs are usually associated with relatively large flares, and 721 of these occurred among the 11,387 events. We selected events that were observed using the Solar Optical Telescope (SOT) [23], 24, 25], 26] onboard Hinode in the visible continuum bands (G-band, blue, green, and red) in the flare-observation mode (taking WL images every $20 \mathrm{~s}$ for each band); 101 were detected. Then, we performed statistical analyses of the hard X-ray data observed by the Reuven Ramaty High Energy Solar Spectroscopic Imager (RHESSI) [27]. Among the 101 events, only one that was observed simultaneously with RHESSI had emission above $1 \mathrm{MeV}$ : the X1.8-class flare on October 23, 2012. We also checked the data from the Gamma-Ray Burst Monitor (GBM) [28] onboard the Fermi spacecraft and found another event with emission above $1 \mathrm{MeV}$ : the M7.9-class flare on June 25, 2015.

We analyzed these two events having WL enhancements using running-difference images of the SOT continuum data. Because there is a possibility - even if the event has no WL enhancements from the SOT data - that WL emission may exist out of the SOT field of view, we also checked the continuum data from the Helioseismic and Magnetic Imager (HMI) [20, [3] onboard the Solar Dynamics Observatory (SDO) [B]]. We found that both events had WL emission observed simultaneously by both instruments.

\subsection{X1.8 flare on October 23, 2012}

As shown in the left-hand panel of Figure प, large Ca II H ribbons were observed in association with an X1.8-class flare on October 23, 2012. WL emission was also clearly observed by Hinode/SOT and SDO/HMI, as shown in the center and right-hand panels of Figure $\mathrm{W}$. WL enhancements were located just under the strong (saturated) Ca II H ribbons. Hard X-ray emission was also observed by RHESSI, with almost the same locations and timings, and gamma-ray emission above $1 \mathrm{MeV}$ with almost the same timing was observed by RHESSI and Fermi/GBM.

The left- and right-hand panels of Figure $\square$ show the gamma-ray spectra observed by RHESSI and Fermi/GBM, respectively. In neither panel could we find clear evidence for $2.2 \mathrm{MeV}$ line emission. From these data, we conclude that line gamma-ray emission is buried in the strong electron bremsstrahlung. From the RHESSI spectra, we found a small enhancement around $2.2 \mathrm{MeV}$, from which we estimated the line gamma-ray flux to be 4.4 photons $/ \mathrm{s} / \mathrm{cm}^{2} / \mathrm{keV}$. Using this value, we estimated the proton flux for the October 23, 2012 event using the magnetic-loop transport and interaction model of Hua et al. [B]]. 


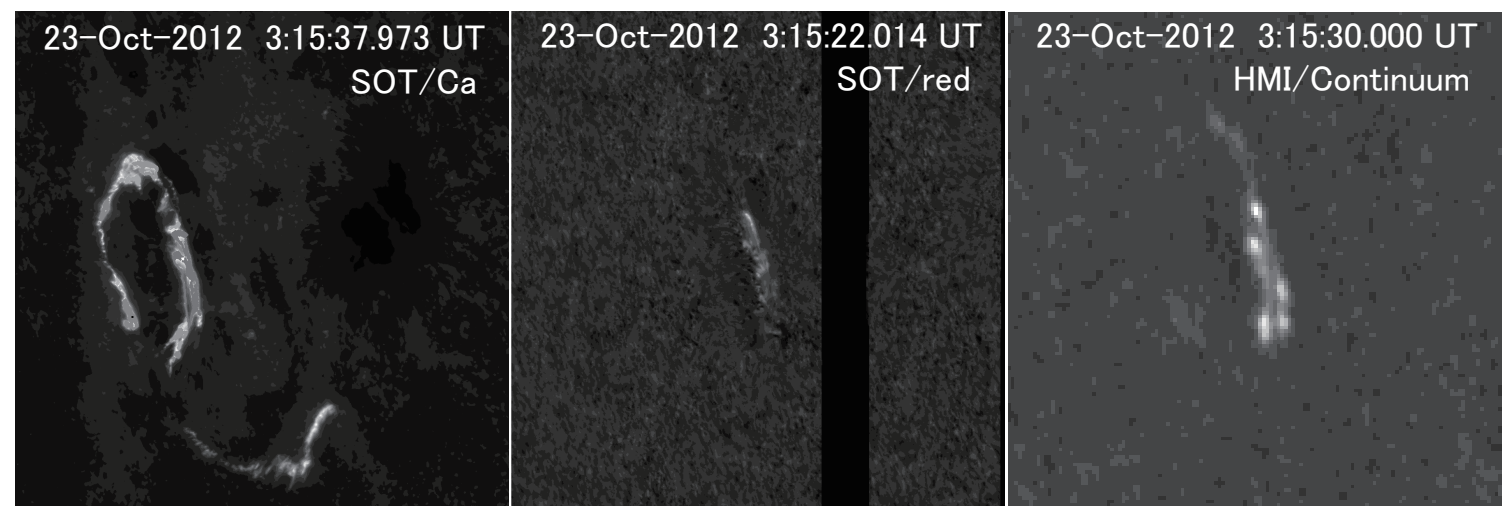

Figure 1: Left: Ca II H image observed by Hinode/SOT. This image was taken in the flare-observation mode (effective spatial resolution of 0.108 " pixel $^{-1}$, with images taken every $20 \mathrm{~s}$ using exposure times of $123 \mathrm{~ms}$ ). Center: Difference image of the Hinode/SOT red continuum band at 3:15:22 UT (effective spatial resolution of 0.108 " pixel $^{-1}$, with images taken every $20 \mathrm{~s}$ ); the image taken at 3:20:06 UT was subtracted to create the difference image. Right: Running-difference images of the continuum band observed by SDO/HMI (effective spatial resolution of 0.6 " pixel $^{-1}$, with images taken every $45 \mathrm{~s}$ ). WL enhancements were observed beginning at 3:14:15 UT.
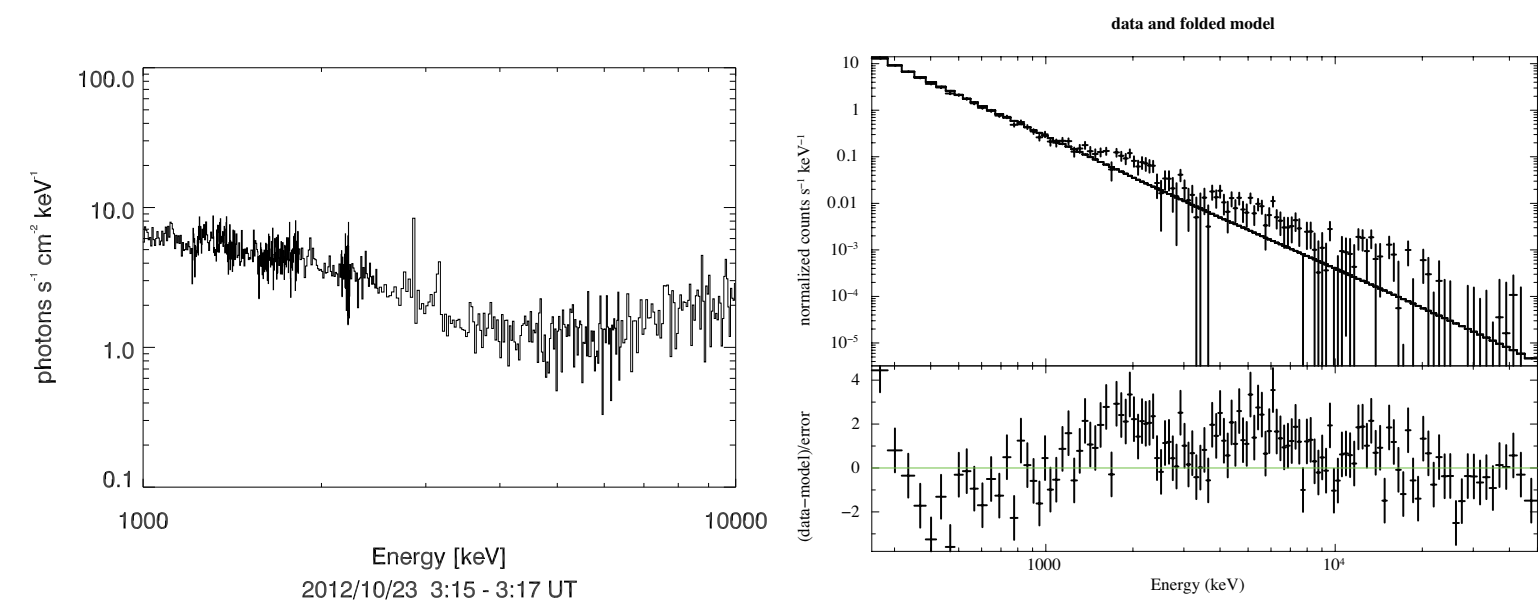

Figure 2: RHESSI (left) and Fermi/GBM (right) spectra of the X1.8 flare on October 23, 2012.

To perform this calculation, it is necessary to set several parameter values such as acceleratedion composition, atmospheric model, flare location, and so on. We did not specify values for "acceleration release time history", "power-law spectral index", or "flare loop length" because it is very difficult to obtain the time profile of the $2.2 \mathrm{MeV}$ line gamma-ray emission. For other parameters, we used observed data or assumed typical values determined from previous observations. For the accelerated ions, we assumed an impulsive-flare composition [33] with $\alpha / \mathrm{p}=0.5$ and ${ }^{3} \mathrm{He} /{ }^{4} \mathrm{He}=1$. For the ambient material, we used the coronal composition of Reames [34], with $\mathrm{He} / \mathrm{H}=0.1$ and $\mathrm{Ne} / \mathrm{O}=0.25$, together with the atmospheric model of Avrett [B5], with photospheric ${ }^{3} \mathrm{He} / \mathrm{H}=3.7 \times 10^{-5}$.

Murphy et al. [B]] estimated the $2.2 \mathrm{MeV}$ line yields per accelerated proton of given energy in Fig. 18 of their paper. They used the pitch-angle scattering mean free path $\lambda=300$ and the 

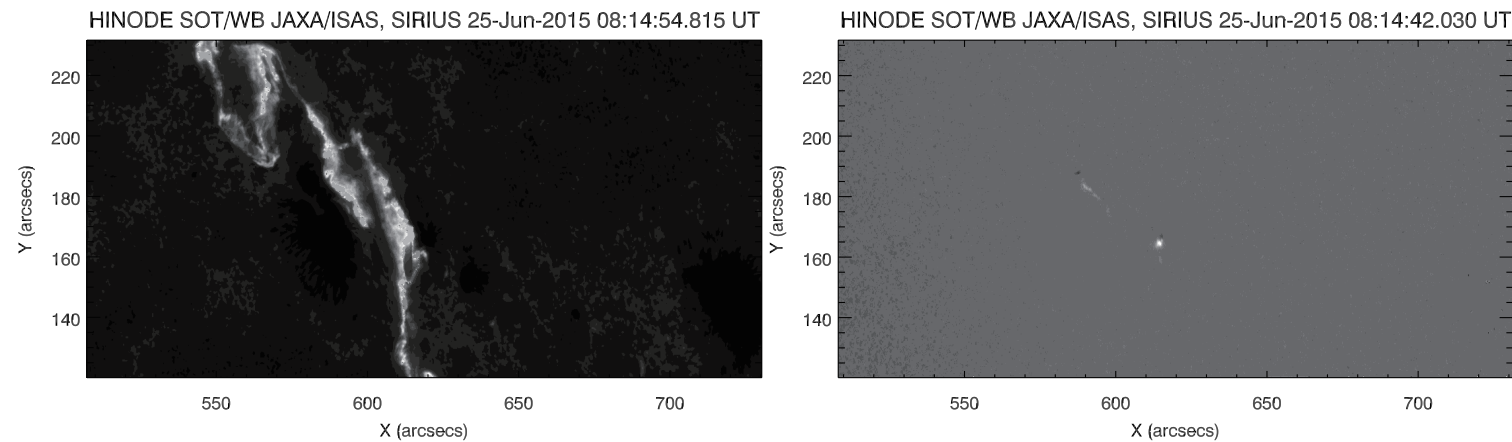

Figure 3: Left: Ca II H image observed by Hinode/SOT (effective spatial resolution of 0.218 " pixel $^{-1}$ ). Right: Difference image of the Hinode/SOT blue continuum band at 8:14:42 UT (effective spatial resolution of 0.218 " pixel ${ }^{-1}$, with images taken every $3 \mathrm{~s}$ ); the image taken $3 \mathrm{~s}$ before was subtracted to form the difference image.

magnetic convergence index $\delta=0.2$ for their calculation. They also used the flare heliocentric angle $\theta_{o b s}=75^{\circ}$. The October 23, 2012 flare occurred at S18E58, and the flare heliocentric angle for this event was $60^{\circ}$. Although there is a difference between these two flare heliocentric angles, the effect of this difference is very small - less than a factor of two in the photon yields.

The mean energy of the original accelerated ions that produce the $2.2 \mathrm{MeV}$ line is $100 \mathrm{MeV}$ [B7]. From Fig. 18 of Murphy et al. [B6], the $2.2 \mathrm{MeV}$ yield ratio was estimated to be $10^{-2}$ photons/sr for $100 \mathrm{MeV}$ ions. From these data, we obtained a total ion energy-production rate of $1.6 \times 10^{28} \mathrm{erg} / \mathrm{s}$.

We also estimated the energy flux of WL emission from the three WL bands of Hinode/SOT. For this calculation, we used the same method as Watanabe et al. [15]. We fitted the data points for the three WL bands using the Planck formula and estimated the temperature of the observed WL emission from this fit. Then, we estimated the energy flux of WL emission from this temperature. This gave the total flux of WL emission at the time of the emission peak (3:15 UT) as $\sim 10^{28} \mathrm{erg} / \mathrm{s}$. As this result is the same order of magnitude as the energy of the accelerated protons, we cannot reject accelerated protons as the possible origin of the WL emission from this flare.

\subsection{M7.9 flare on June 25, 2015}

Large Ca II H ribbons were observed in association with an M7.9-class flare on June 25, 2015, as shown in the left-hand panel of Figure [3. WL emission was also clearly observed by Hinode/SOT (as shown in the right-hand panel of Figure [3) and SDO/HMI (not shown here). These WL enhancements were also located just under the strong (saturated) $\mathrm{Ca}$ II $\mathrm{H}$ ribbons. Unfortunately, RHESSI was on the night side of the Earth during this flare. On the other hand, Fermi/GBM did observe gamma-ray emission from this flare at energies above $1 \mathrm{MeV}$.

Figure 7 shows the gamma-ray spectra observed by Fermi/GBM. In this figure, we found weak evidence for $2.2 \mathrm{MeV}$ line emission. Although we could not estimate the $2.2 \mathrm{MeV}$ line gamma-ray flux without the RHESSI data, the strength of the gamma-ray emission observed by Fermi/GBM was two orders of magnitude smaller than that from the October 23, 2012 event.

We roughly estimated the accelerated-proton flux using the same method as for the October 23, 2012 event. This flare occurred at N09W42, and the flare heliocentric angle for this event was 


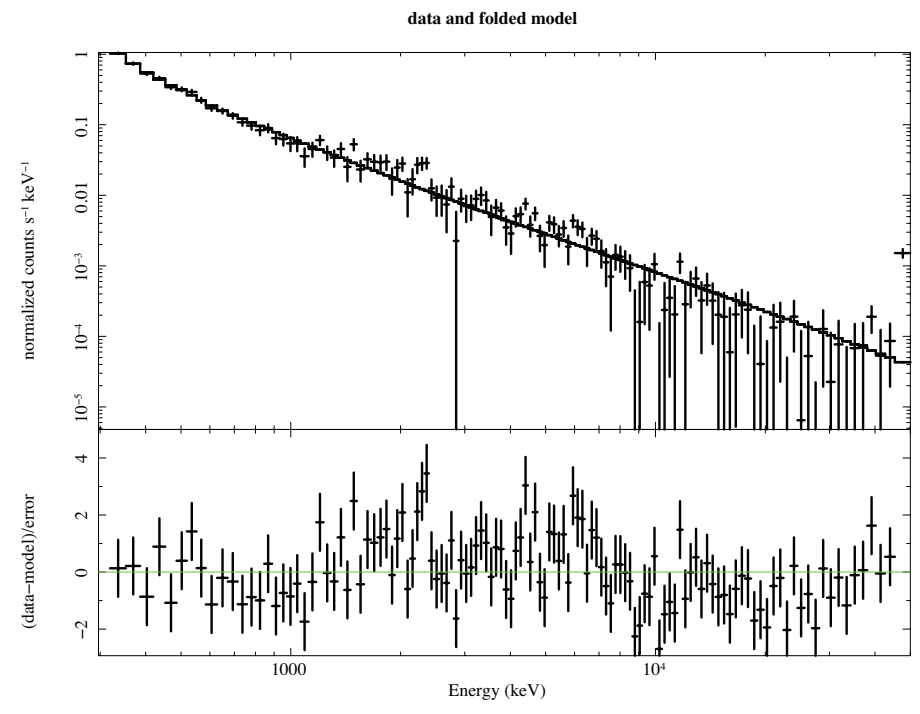

Figure 4: Fermi/GBM spectrum of the M7.9 flare on June 25, 2015.

$42^{\circ}$. Accordingly, the $2.2 \mathrm{MeV}$ line yield per accelerated proton may be as much as twice that of the October 23, 2012 event. Assuming the $2.2 \mathrm{MeV}$ line gamma-ray flux for the June 25, 2015 event to be two orders of magnitude smaller than that for the October 23, 2012 event, we estimated the total accelerated-ion energy rate to be $\sim 10^{26} \mathrm{erg} / \mathrm{s}$.

As the June 25, 2015 event was observed only in the blue band of Hinode/SOT, we could not estimate the temperature distribution for this event. We therefore assumed the peak intensity of the blue band to be correlated with the WL flux and estimated the WL emission flux from this. We estimated the peak intensities of the October 23, 2012 and June 25, 2015 events to be $6.7 \times 10^{5}$ and $1.5 \times 10^{5} \mathrm{DN} / \mathrm{s}$, respectively. The peak intensity of the October 23,2012 event is thus more than four times larger than that of the June 25, 2015 event. Using this factor of four, we estimate the WL emission flux for the June 25,2015 event to be $2 \times 10^{27} \mathrm{erg} / \mathrm{s}$. For this case, the accelerated-ion energy is not sufficient to explain the WL emission flux. We can thus reject accelerated protons as the origin of the WL emission associated with this flare.

\section{Discussion and summary}

WL emission was clearly observed by Hinode/SOT and SDO/HMI in association with an X1.8class flare on October 23, 2012 and with an M7.9 class flare on June 25, 2015. In both events, gamma-ray emission above $1 \mathrm{MeV}$ was also observed by Fermi/GBM and/or RHESSI. Although we could not find evidence for the $2.2 \mathrm{MeV}$ neutron-capture line emission in the October 23, 2012 event, the June 25, 2015 event has a small enhancement around $2.2 \mathrm{MeV}$. The strength of the gamma-ray emission around $2.2 \mathrm{MeV}$ for the June 25, 2015 event is two orders of magnitude smaller than that for the October 23, 2012 event. We estimated the total energy of accelerated protons and the intensity ratio for each event using the Hua et al. (2002) magnetic-loop transport and interaction model [B2]. The total accelerated-ion energy rates for the October 23, 2012 event and the June 25,2015 event are estimated to be $1.6 \times 10^{28}$ and $\sim 10^{26} \mathrm{erg} / \mathrm{s}$, respectively. 
The total energy of WL emission can also be estimated by fitting the three color bands of Hinode/SOT and the intensity ratio of peak emission. The total flux of WL emission for the October 23, 2012 event is estimated to be $\sim 10^{28} \mathrm{erg} / \mathrm{s}$ and that for the June 25, 2015 event is estimated to be $2 \times 10^{27} \mathrm{erg} / \mathrm{s}$. Although the total accelerated-ion energy for the October 23, $2012 \mathrm{event}$ is sufficient to produce the observed WL emission, we cannot explain the WL emission flux for the June 25, 2015 event from the accelerated-proton energy.

Figures $\square$ and $\$$ show the time-integrated gamma-ray energy spectra, and we used the timeintegrated WL emission strength to make the energy comparison. WL emission for the October 23, 2012 event continued for $4 \mathrm{~min}$, and that for the June 25, 2015 event continued for $1.5 \mathrm{~min}$. When we estimated the time-integrated WL (blue) intensity for the October 23, 2012 event and for the June 25, 2015 event, we obtained $8.0 \times 10^{7}$ and $6.8 \times 10^{6} \mathrm{DN}$, respectively. The difference is just one order of magnitude, but the WL emission energy for the June 25, 2015 event still cannot be explained by the accelerated-ion energy.

We performed statistical analyses of WLFs using Hinode/SOT and found that more than 50 events have WL enhancements. However, only two events were accompanied by gamma-ray emission above $1 \mathrm{MeV}$ and did not depend on the strength of the WL emission. This fact also indicates that WL emission does not originate mainly from accelerated ions.

\section{Acknowledgments}

Hinode is a Japanese mission developed and launched by ISAS/JAXA, with NAOJ as domestic partner and NASA and STFC (UK) as international partners. It is operated by these agencies in co-operation with ESA and NSC (Norway). This work was supported by JSPS KAKENHI Grant Numbers JP15K17622, JP16H01187. This work was carried out by the joint research program of the Institute for Space-Earth Environmental Research (ISEE), Nagoya University.

\section{References}

[1] Carrington, 1859, MNRAS, 20, 13

[2] Matthews, S. A., van Driel-Gesztelyi, L., Hudson, H. S., Nitta, N. V. 2003, AAP, 409, 1107

[3] Hudson, H. S., Wolfson, C. J., \& Metcalf, T. R. 2006, Solar Physics, 234, 1, 79

[4] Jess, D. B., Mathioudakis, M., Crockett, P. J., Keenan, F. P. 2008, ApJL, 688, 2, L119

[5] Wang et al. 2009, Res. Astron. Astrophys., 9, 127

[6] Neidig, D. F. 1989, Solar Physics, 121, 261

[7] Ding, M. D., Liu, Y., Yeh, C.-T., Li, J. P. 2003, AAP, 403, 1151

[8] Fletcher, L., Hannah, I. G., Hudson, H. S., and Metcalf, T. R. 2007, ApJ, 656, 2, 1187

[9] Watanabe, K., Krucker, S., Hudson, H., et al. 2010, ApJ, 715, 1, 651

[10] Krucker, S., Hudson, H. S., Jeffrey, N. L. S., et al. 2011, ApJ, 739, 2, 96

[11] Kuhar, M., Krucker, S., Martínez Oliveros, J.-C., Battaglia, M., Kleint, L. 2016, ApJ, 816:6

[12] Battaglia, M. \& Kontar, E. P. 2011, AAP, 533, L2 
[13] Martínez Oliveros, J.-C., Hudson, H. S., Hurford, G. J., et al. 2012, ApJL, 753, 2, L26

[14] Battaglia, M. \& Kontar, E. P. 2012, ApJ, 760, 2, 142

[15] Watanabe, K., Shimizu, T., Masuda, S., et al. 2013, ApJ, 776, 123

[16] Krucker, S., Saint-Hilaire, P., Hudson, H. S., et al. 2015, ApJ, 802, 19

[17] Svestka, Z. 1970, Solar Physics, 13, 2, 471

[18] Biermann, V. L., et al. 1951, Z. Naturforsch., 6, 47

[19] Watanabe, K., Muraki, Y., Matsubara, Y., et al. 2003, ApJ, 592, 590

[20] Watanabe, K., Gros, M., Stoker, P. H., et al. 2006, ApJ, 636, 1135

[21] Sako, T., Watanabe, K., Muraki, Y., et al. 2006, ApJL, 651, L69

[22] Watanabe, K., Masuda, S., and Segawa, T. 2012, Solar Physics, 279, 1, 317

[23] Tsuneta, S., Suematsu, Y., Ichimoto, K., et al. 2008, Solar Physics, 249, 2, 167

[24] Suematsu, Y., Tsuneta, S., Ichimoto, K., et al. 2008, Solar Physics, 249, 2, 197

[25] Shimizu, T., Nagata, S., Tsuneta, S., et al. 2008, Solar Physics, 249, 2, 221

[26] Ichimoto, K., Lites, B., Elmore, D., et al. 2008, Solar Physics, 249, 2, 233

[27] Lin, R. P., Dennis, B. R., Hurford, G. J., et al. 2002, Solar Physics, 210, 1, 3

[28] Meegan, C., Lichti, G., Bhat, P. N., et al. 2009, ApJ, 702, 791

[29] Scherrer, P. H., Schou, J., Bush, R. I., et al. 2012, Solar Physics, 275, 207

[30] Schou, J., Scherrer, P. H., Bush, R. I., et al. 2012, Solar Physics, 275, 229

[31] Pesnell, W. D., Thompson, B. J., \& Chamberlin, P. C. 2012, Solar Physics, 275, 3

[32] Hua, X.-M., Kozlovsky, B., Lingenfelter, R.E., et al. 2002, Astrophys. J. Suppl. Series, 140, 563

[33] Ramaty, R., Mandzhavidze, N., \& Kozlovsky, B. 1996, AIP Conf. Proc. 374, High Energy Solar Physics, ed. R. Ramaty, N. Mandzhavidze, \& X.-M. Hua (Woodbury: AIP), 172

[34] Reames, D.V. 1995, Adv. Sp. Res., 15, No. 7, 41

[35] Avrett, E.H. 1981, The Physics of Sunspots, ed. L.E. Cram and J.H. Thomas, (Sunspot: Sacramento Peak Obs.), 235

[36] Murphy, R. J., Kozlovsky, B., Share, G. H., et al. 2007, Astrophys. J. Suppl. Series, 168, 167

[37] Lockwood, J. A., Debrunner, H., Ryan, J. M. 1997, Solar Physics, 173, 1, 151 